Classification

Physics Abstracts

$61.80 \mathrm{M}$

\title{
A zone spectrum of the ultrarelativistic channelled particles in a crystal
}

\author{
B. A. Chevganov and I. D. Feranchuk \\ Department of Physics, Byelorussian State University, Minsk-80, USSR
}

(Reçu le 23 septembre 1981, révisé le 8 juin 1982, accepté le 27 juillet 1982)

\begin{abstract}
Résumé. - On obtient l'équation de dispersion qui définit le spectre d'énergie de bande du mouvement transverse de particules chargées canalisées dans un plan. La solution numérique de cette équation est obtenue en utilisant le potentiel réel du plan cristallographique. On calcule les coefficients de population des niveaux d'énergie et on compare les solutions classique et quantique du problème de la canalisation des particules.
\end{abstract}

\begin{abstract}
The dispersion equation which defines the band energy spectrum of the transverse movement of the plane channelled charge particle is obtained in this work. The numerical solution of this equation is found using the real crystallographic plane potential. The population coefficients of the energy levels are calculated and quantum and classical solutions of the problem of particle channelling are compared.
\end{abstract}

1. Introduction. - The possibility of infrared photon formation under transitions between transverse energy levels of the channelled protons was at first mentioned by Tomson [1]. In references [2,3] this process was considered for channelled electrons and for the optical range of the radiated photons. However, the interest in the problem of channelling radiation was largely stimulated by the works of Baryshevsky and Dubovskaya [4] and Kumakhov [5] where they at first showed that because of the Doppler effect ultrarelativistic electrons and positrons would radiate $\mathrm{X}$-rays and photons in harder range. In reference [4] the quantum theory in which the channelling radiation was caused by transitions between energy zones of the transverse movement of the channelled particle was given and in reference [5] the channelled particle was considered as a radiating classical oscillator.

The radiation from the channelled particles was discovered by several experimental groups [6-8] and at present the quantitative theoretical description of the real radiation spectrum is a very actual problem. Such analysis is necessary to choose the optimal conditions for obtaining the intensive X-ray bunches so as to use the above-mentioned radiation for studying of crystal structures. In many works devoted to the theory of the channelling radiation (see, for example, reference [9] and cited works) the later was considered as a radiation under transitions between below-barrier isolated energy levels only. But in accordance with the accurate quantum theory described in references [10-11] the quantitative description of the experimental spectrum can be obtained if all the possible transitions between energy zones both below-barrier and above-barrier are taken into consideration.

In the present work the quantum calculations of the electrons and positrons energy band spectrums and of the population coefficients are fulfilled for the case of plane channelling. The main results were briefly presented earlier [12]. Our calculations are based on the quasiclassical approximation. As was shown in references [13-14] the correct utilization of this approximation enables account to be taken of effects of the below-barrier passage and the above-barrier reflection. Using this method we have built the simple and sufficiently accurate algorithm for the numerical calculation of the band spectrums and wave functions in the whole range of the transverse energy of the channelled particles. Our calculation method is most convenient when the total particle energy

$$
E \gtrsim 5-10 \mathrm{MeV} \text {. }
$$

The analogous problem was independently investigated by Tulupov [15]. But the method of many-wave diffraction theory used in this work is not efficient for numerical calculation of the band spectrum in the case of sufficiently large total energy of the channelled particle and for calculation of high above-barrier zones whose population are essential if the angle dispersion of the particles in the real beams is taken into account. Therefore our results and the results represented in reference [15] are not covered in fact. 
As will be shown further, our calculation method possesses additional advantages in comparison with the direct solution of the Schrödinger equation. These advantages are connected first with the possibility of zone spectrum "scaling" and secondly due to simple way of population coefficients averaging over the angle dispersion in the particle beam.

2. A zone spectrum of the one-dimension crystal in the quasiclassical approximation. - It is well known that in the plane channelling case the ultrarelativistic $(E \gg m)$ particle movement can be described by the equation which formally coincides with non-relativistic one-dimension Schrödinger equation $(\hbar=c=1)$

$$
\left[-\frac{1}{2 E_{0}} \frac{\mathrm{d}^{2}}{\mathrm{~d} x^{2}}+V(x)\right] \varphi(x)=\varepsilon \varphi(x),
$$

where $E_{0}=\sqrt{p_{z}^{2}+p_{y}^{2}+m^{2}}$ is the longitudinal particle energy $\left(p_{z}\right.$ and $p_{y}$ are the momentum components, $m$ is the particle mass); $Z$-axis coincides with the particle velocity direction; $X$-axis is perpendicular to the channel plane; $V(x)$ is the potential of the crystallographic plane, which can be built as follows [16] :

$$
V(x)=\left.\sum_{\tau_{x}} g(\tau)\right|_{\tau_{y}=\tau_{z}=0} \mathrm{e}^{i \tau_{x} x} ; \quad V(x+d)=V(x) .
$$

Here $g(\tau)$ are the Fourier components of the accurate three-dimensional potential of the particle-crystal interaction; these components include the DebyeWaller factor, which appears when potential averaging over the atoms thermal vibrations; $d$ is the lattice period along the $X$-axis; $\tau$ is the reciprocal lattice vector.

The total energy $E$ and the wave function $\psi$ of the particle are connected with the transverse energy $\varepsilon$ and with the function $\varphi(x)$ as follows

$$
\begin{gathered}
E \approx E_{0}+\varepsilon\left(E_{0}\right) \\
\psi(\mathbf{r})=\mathrm{e}^{i\left(p_{z} z+p_{y} y\right)}\left(1-\frac{i}{2 E_{0}} \alpha_{x} \frac{\mathrm{d}}{\mathrm{d} x}\right) \varphi(x) u(\mathbf{p}),
\end{gathered}
$$

where $\alpha_{x}$ is the Dirac matrix ; $u(\mathbf{p})$ is the spinor describing a free particle with momentum $\mathbf{p}$. The expressions (3) are fulfilled with accuracy $\sim\langle V\rangle / E_{0}$.

Thus we come to the problem of building the energy band spectrum in the one-dimensional periodical potential. The general dispersion equation determining the energy spectrum can be found without any approximation [14]. Really, there are two linear independent solutions of the equation (1) in the region where $\varepsilon>V(x)$. Let us call these solutions $f(x)$ and $f^{*}(x)$. Then in the region $k d \leqslant x \leqslant(k+1) d ; k=0,1,2, \ldots$ the general solution of the equation (1) may be written as follows :

$$
\varphi_{k}(x)=C_{1} f(x)+C_{2} f^{*}(x) .
$$

In the region $(k+1) d \leqslant x \leqslant(k+2) d$ each function $f$ and $f^{*}$ transforms into some linear combination of the same functions, that is

$$
\begin{aligned}
f(x+d) & =D f(x)+R f^{*}(x) \\
f^{*}(x+d) & =D^{*} f^{*}(x)+R^{*} f(x),
\end{aligned}
$$

and coefficients $R$ and $D$ satisfy the condition $|D|^{2}=1+|R|^{2}$, which follows from the condition of the flow periodicity. Then

$$
\begin{aligned}
\varphi_{k+1}(x)=\varphi_{k}(x+d)= & C_{1}\left(D f+R f^{*}\right)+ \\
& +C_{2}\left(D^{*} f^{*}+R^{*} f\right),
\end{aligned}
$$

and in accordance with the Bloch theorem

$$
\varphi_{k+1}(x)=\mathrm{e}^{i \kappa d} \varphi_{k}(x),
$$

where $\kappa$ is the crystal momentum. As the functions $f$ and $f^{*}$ are linearly independent, the coefficients $C_{1}$ and $C_{2}$ must satisfy the system of the equations

$$
\begin{aligned}
& C_{1} D+C_{2} R^{*}=C_{1} \mathrm{e}^{i \kappa d} \\
& C_{1} R+C_{2} D^{*}=C_{2} \mathrm{e}^{i \kappa d} .
\end{aligned}
$$

The condition of the existence of a non-trivial solution of the system (7) leads to the dispersion equation

$$
\cos \kappa d=|D| \cos \varphi(\varepsilon)
$$

where

$$
D(\varepsilon) \equiv|D(\varepsilon)| \mathrm{e}^{i \varphi(\varepsilon)} .
$$

Let us introduce the reflection coefficient $R_{1}$ and the passage coefficient $D_{1}$ instead of $R$ and $D$. The new coefficients are defined in the usual manner. For example in the case of a wave passing over the potential barrier from left to right one can find the following relation

$$
\begin{gathered}
D=\frac{1}{D_{1}} ; \quad R=\frac{R_{1}}{D_{1}} ; \quad D_{1}=\left|D_{1}\right| \mathrm{e}^{i \varphi_{1}(\varepsilon)} ; \\
\cos \kappa d=\frac{1}{\left|D_{1}\right|} \cos \varphi_{1}(\varepsilon) .
\end{gathered}
$$

In order to find the analytical expression for $D_{1}$ it is necessary to use some approximations for calculating the functions $f$ and $f^{*}$. In the present work we utilize the quasiclassical approximation. For the equation (1) this approximation accuracy is defined by the following parameter

$$
\xi=\frac{1}{E_{0} V_{\mathrm{m}} d^{2}} \approx \frac{1}{n^{2}},
$$

where $n$ is the number of discrete spectrum levels in the isolated potential pit coinciding with the channel potential; $V_{\mathrm{m}}$ is the maximum value of the crystallo- 
graphic plane potential. In this approximation $f$ and $f^{*}$ are defined by the following expressions

$$
f(x)=\frac{\exp \left(i \int p(x) \mathrm{d} x\right)}{\sqrt{p(x)}} ; f^{*}=\frac{\exp \left(-i \int p(x) \mathrm{d} x\right)}{\sqrt{p(x)}} ;
$$

$$
p(x)=\sqrt{2 E_{0}[\varepsilon-V(x)]} .
$$

In the region $\varepsilon<V_{\mathrm{m}}$ coefficient $D_{1}$ is defined by the well known expression

$$
\left|D_{1}\right| \approx \mathrm{e}^{-\tau_{1}} ;\left|R_{1}\right| \approx 1 ; \varphi_{1}(\varepsilon)=\sigma_{1}(\varepsilon),
$$

provided that $\left|D_{1}\right| \ll 1$.

Here

$$
\begin{aligned}
& \sigma_{1}=\int_{x_{1}}^{x_{2}} \sqrt{2 E_{0}[\varepsilon-V(x)]} d x ; \\
& \tau_{1}=\int_{x_{2}}^{x_{3}} \sqrt{2 E_{0}[V(x)-\varepsilon]} d x
\end{aligned}
$$

and $x_{1} ; x_{2} ; x_{3}$ are the turning points; energy $\varepsilon$ is counted off the potential minimum.

As distinct from the classical case the reflection coefficient is not equal zero when $\varepsilon>V_{\mathrm{m}}$. In the quasiclassical approximation the method of the calculation of $D_{1}$ for $\varepsilon>V_{m}$ was developed in reference [13]. This method is based on using the special countour integration to build the functions $f$ and $f^{*}$. This countour must pass over complex turning points which are defined by the equality

$V\left(z_{0}^{k}\right)=\varepsilon \quad$ and $\quad z_{0}^{k}=z_{0}+k d ; \quad k=0,1,2, \ldots$

Not repeating the calculations in reference [13], we cite the expression for the reflection coefficient. In this case

$$
R_{1}=\exp \left\{i \int_{z_{0}^{k}}^{z_{0}^{k+1}} \sqrt{2 E_{0}[\varepsilon-V(z)]} \mathrm{d} z\right\}
$$

If the function $V(x)$ is symmetrical with respect to point $x=d / 2$ (this condition is usually fulfilled for a real potential) then the integral countour may be chosen in such a manner that the values $\left|D_{1}\right|$ and $\varphi_{1}(\varepsilon)$ are expressed by the real integrals. Then

$$
\begin{gathered}
\varphi_{1}(\varepsilon)=\sigma_{0}(\varepsilon)=\int_{-d / 2}^{d / 2} \sqrt{2 E_{0}[\varepsilon-V(x)]} \mathrm{d} x ; \\
\left|D_{1}\right|=1-\frac{1}{2} \mathrm{e}^{-\tau_{2}} ; \\
\tau_{2}=4 \int_{0}^{y_{0}} \sqrt{2 E_{0}\left[\varepsilon-V\left(\frac{d}{2}+i y\right)\right]} \mathrm{d} y ; \\
z_{0}=\frac{d}{2}+i y_{0} .
\end{gathered}
$$

The expressions (12) and (14) fail when the transversal movement energy is approximately equal to the barrier potential $V_{\mathrm{m}}$. Therefore we shall obtain the formula which will apply when $\varepsilon \sim V_{\mathrm{m}}$ and which reduces to (12) or (14) in the corresponding limit cases. For the case $\varepsilon>V_{\mathrm{m}}$ the analogous investigation was carried out in reference [17] in connection with another problem.

Near the barrier potential

$$
V(x) \approx V_{\mathrm{m}}-K x_{1}^{2} ; \quad K=\frac{1}{2}\left|V^{\prime \prime}\left(\frac{d}{2}\right)\right| ; \quad x_{1} \equiv x-\frac{d}{2}
$$

and the equation (1) has the analytical solution

$$
\begin{gathered}
\varphi(x)=A_{1} D_{m}\left[x_{1} \exp \left(-i \frac{\pi}{4}\right) \sqrt[4]{4 \lambda}\right]+B_{1} D_{-m-1}\left[x_{1} \exp \left(i \frac{\pi}{4}\right) \sqrt[4]{4 \lambda}\right] \\
\lambda=2 K E_{0} ; \quad m=-\frac{1}{2}-i \sqrt{\lambda} x_{0}^{2} ; \quad x_{0}^{2}=-\frac{\varepsilon-V_{\mathrm{m}}}{K^{2}}
\end{gathered}
$$

where $D_{m}(z)$ is the parabolic cylinder function.

To the right of the potential barrier, that is when $x_{1}>0, D_{m}$ and $D_{-m-1}$ turn into the quasiclassical functions $f$ and $f^{*}$ respectively in the asymptotic limit. Let us count $B_{1}=0$ as we are interested by the reflection and passage coefficients on the solitary barrier. Then for $x_{1} \sqrt[4]{4 \lambda} \gg 1$

$$
\varphi(x) \approx \frac{A_{1}}{\sqrt[4]{4 \lambda x_{1}^{2}}} \exp \left[i\left(\frac{\sqrt{\lambda}}{2} x_{1}^{2}-\frac{\sqrt{\lambda}}{2} x_{0}^{2} \ln x_{1} \sqrt[4]{4 \lambda}-i \frac{\pi}{8}-i \frac{\pi}{8} \sqrt{\lambda} x_{0}^{2}\right)\right] \approx \frac{A^{\prime}}{\sqrt{p(x)}} \exp \left(i \int_{0}^{x_{1}} p(x) \mathrm{d} x\right) .
$$

In the region $x_{1}<0$ the function $\varphi(x)$ has the next form [18]

$$
\begin{aligned}
\varphi(x)=A_{1} D_{m}[\sqrt[4]{4 \lambda} & \left.x_{1} \exp \left(i \frac{3 \pi}{4}\right)\right] \approx \\
& \approx A_{1}\left\{D_{m}\left[\sqrt[4]{4 \lambda} x_{1} \exp \left(-i \frac{\pi}{4}\right)\right]-\frac{\sqrt{2 \pi}}{\Gamma(-m)} \mathrm{e}^{i \pi m} D_{-m-1}\left[\sqrt[4]{4 \lambda} x_{1} \exp \left(i \frac{\pi}{4}\right)\right]\right\},
\end{aligned}
$$


and when $\left|x_{1} \sqrt[4]{4 \lambda}\right| \gg 1$, coincides with the linear combination of the functions $f$ and $f^{*}$

where

$$
\varphi(x)=A \frac{\exp \left(i \sigma_{0}-i \int_{0}^{x_{1}} p(x) \mathrm{d} x\right)}{\sqrt{p(x)}}+B \frac{\exp \left(-i \sigma_{0}+i \int_{0}^{x_{1}} p(x) \mathrm{d} x\right)}{\sqrt{p(x)}},
$$

$$
\frac{A^{\prime}}{A}=D_{1} ; \quad \frac{B}{A}=R_{1} .
$$

Using the well known asymptotic expressions for the functions $D_{m}$ and $D_{-m-1}[18]$ one can find

$$
\begin{aligned}
& D_{1}=\frac{\Gamma\left(\frac{1}{2}+\frac{i}{2} \sqrt{\lambda} x_{0}^{2}\right)}{\sqrt{2 \pi}} \exp \left(i \sigma_{0}-\frac{\pi}{4} \sqrt{\lambda} x_{0}^{2}\right) ; \\
& R_{1}=\frac{\Gamma\left(\frac{1}{2}+\frac{i}{2} \sqrt{\lambda} x_{0}^{2}\right)}{\sqrt{2 \pi}} \exp \left(2 i \sigma_{0}-i \frac{\pi}{4}+\frac{\pi}{4} \sqrt{\lambda} x_{0}^{2}\right),
\end{aligned}
$$

where $\Gamma(z)$ is the $\Gamma$-function.

Thus when $\varepsilon$ is near barrier potential

$$
\begin{gathered}
\left|D_{1}\right|=\frac{\left|\Gamma\left(\frac{1}{2}+\frac{i}{2} \sqrt{\lambda} x_{0}^{2}\right)\right|}{\sqrt{2 \pi}} \exp \left(-\frac{\pi}{4} \sqrt{\lambda} x_{0}^{2}\right)=\frac{\exp \left(-\frac{\pi}{2} \sqrt{\lambda} x_{0}^{2}\right)}{2 \cosh \frac{\pi}{2} \sqrt{\lambda} x_{0}^{2}} \\
\varphi_{1}(\varepsilon)=\arg D_{1}=\sigma_{0}+\gamma ; \quad \gamma=\arg \Gamma\left(\frac{1}{2}+i \sqrt{\lambda} x_{0}^{2}\right)
\end{gathered}
$$

Now we take into account that in the region where the solution (15) may be used the following equality takes place

$$
\begin{array}{ll}
\pi \sqrt{\lambda} x_{0}^{2}=\int_{x_{2}}^{x_{3}} \sqrt{2 E_{0}[V(x)-\varepsilon]} \mathrm{d} x=\tau_{1} ; & \varepsilon<V_{\mathrm{m}} \\
\pi \sqrt{\lambda} x_{0}^{2}=-\int_{0}^{y_{0}} \sqrt{2 E_{0}\left[\varepsilon-V\left(\frac{d}{2}+i y\right)\right]}=-\tau_{2} ; & \varepsilon>V_{\mathrm{m}} .
\end{array}
$$

As a result we obtain the dispersion equation in the following form

$$
\begin{array}{ll}
\cos \kappa d=2 \mathrm{e}^{\tau_{1} / 2} \cosh \frac{\tau_{1}}{2} \cos \sigma ; & \varepsilon \leqslant V_{\mathrm{m}} ; \\
\cos \kappa d=2 \mathrm{e}^{-\tau_{2} / 2} \cosh \frac{\tau_{2}}{2} \cos \sigma_{0} ; & \varepsilon>V_{\mathrm{m}},
\end{array}
$$

which permits us to build the zone spectrum in the whole range of the transverse particle energy.

Let us consider the limit cases when the solution of the equation (19) may be obtained analytically.

a) $\varepsilon<V_{\mathrm{m}} ; \tau_{1} \gg 1$;

$$
\begin{gathered}
\sigma=\pi\left(n+\frac{1}{2}\right)+\mathrm{e}^{-\tau_{1}} \cos \kappa d, \quad n=0,1,2, \ldots \\
\varepsilon_{n \kappa} \approx \varepsilon_{n}^{(0)}+\Delta \varepsilon_{n \kappa},
\end{gathered}
$$

where $\varepsilon_{n}^{(0)}$ coincides with the energy of the discrete spectrum levels in the isolated potential pit

$$
\int_{x_{1}}^{x_{2}} \sqrt{2 E_{0}\left[\varepsilon_{n}^{(0)}-V(x)\right]} \mathrm{d} x=\pi\left(n+\frac{1}{2}\right)
$$


and

$$
\Delta \varepsilon_{n \kappa} \approx \mathrm{e}^{-\tau_{1}\left(\varepsilon_{n}^{(0)}\right)} \cos \kappa d \ll \varepsilon_{n+1}^{(0)}-\varepsilon_{n}^{(0)}
$$

defines the value of allowed energy band is significantly less than the distance between bands.

b) $\varepsilon>V_{\mathrm{m}} ; \tau_{2} \gg 1 ; \cos \kappa d \approx\left(1+\mathrm{e}^{-\tau_{2}}\right) \cos \sigma$,

$$
\begin{gathered}
\int_{-d / 2}^{d / 2} \sqrt{2 E_{0}\left[\varepsilon_{n \kappa}-V(x)\right]} \mathrm{d} x=\pi n+\kappa d+\delta_{n}(\kappa) ; \quad \delta_{n} \ll 1 ; \quad 0 \leqslant \kappa d \leqslant \pi ; \\
\delta_{n}(\kappa)=(-1)^{n} \kappa_{1} \pm \sqrt{\kappa_{1}^{2}+2 \mathrm{e}^{-\tau_{2}}} ; \quad \kappa_{1}=\pi-\kappa d .
\end{gathered}
$$

In this case the energy spectrum consists of wide allowed energy bands and narrow forbidden energy bands. The width of the forbidden energy band is expressed as follows

$$
\Delta \varepsilon_{n \kappa} \approx 2 \sqrt{2 \mathrm{e}^{-\tau_{2}\left(\varepsilon_{n \kappa}\right)}}
$$

and for $\varepsilon \gg V_{\mathrm{m}}$ we have

$$
\varepsilon_{n \kappa} \approx \frac{(\pi n+\kappa d)^{2}}{2 E_{0} d^{2}} .
$$

The exact solution of equation (19) for the Si crystal has been obtained numerically. The parameters of the considered problem have been defined by the conditions of the experiment [8]. The potential used as the interplane potential has been obtained in Moliere's approximation with averaging over the thermal vibrations [19]. In the case of positrons this potential can be written as

$$
V_{\mathrm{e}^{+}}=E_{0} \psi_{a}^{2} \sum_{i=1}^{3} \frac{1}{2} \gamma_{i} \mathrm{e}^{\tau_{i}}\left\{\exp \left(-\beta_{i} \frac{x}{a}\right) \operatorname{erfc}\left[\frac{1}{\sqrt{2}}\left(\frac{\beta_{i} u_{1}}{a}-\frac{x}{u_{1}}\right)\right]+\exp \left(\beta_{i} \frac{x}{a}\right) \operatorname{erfc}\left[\frac{1}{\sqrt{2}}\left(\frac{\beta_{i} u_{1}}{a}+\frac{x}{u_{1}}\right)\right]\right\}
$$

and in the case of electrons the potential is expressed as follows

$$
V_{\mathrm{e}^{-}}(x)=-V_{\mathrm{e}^{+}}\left(x-\frac{d}{2}\right)+V_{\mathrm{m}} \text {. }
$$

The definition of the potential parameters $\psi_{a} ; \gamma_{i} ; \beta_{i} ; \tau_{i} ; u_{1}$ are given in reference [19].

Using the optimal program permits us to build the whole energy spectrum with a precision of $1 \%$ both for electrons and for positrons in several minutes on a computer ES 1033.

As for example figure 1 shows the energy spectra of electrons and positrons which have the same total energy $E_{0}=28,56 \mathrm{MeV}$ and channel along the $(1,1,0)$ crystallographic plane in Si crystal.

3. The wave function normalization and the population coefficients of the energy levels. - According to formulae (4) and (7) the stationary wave function of the channelled particle is expressed as

where

$$
\varphi(x)=C_{1} f(x)+C_{2} f^{*}(x),
$$

$$
C_{2}=q(\varepsilon) C_{1}, \quad q(\varepsilon)=\frac{D_{1}(\varepsilon) \mathrm{e}^{i \kappa d}-1}{R_{1}} .
$$

The factor $C_{1}$ is determined by the condition of the wave function normalization

$$
\left|C_{1}\right|^{2}\left(1+|q|^{2}\right) I=1 ; \quad I=\int_{-d / 2}^{d / 2}|f(x)|^{2} \mathrm{~d} x .
$$

we have neglected the contribution of fast-oscillating functions $f^{2}$ and $f^{* 2}$ in the integral.

If the energy levels are far from the barrier potential the factor $I$ is expressed as follows

$$
I=\frac{T(\varepsilon)}{E_{0}}= \begin{cases}\int_{x_{1}}^{x_{2}} \frac{\mathrm{d} x}{p(x)} ; & \varepsilon<V_{\mathrm{m}} \\ \int_{-d / 2}^{d / 2} \frac{\mathrm{d} x}{p(x)} ; & \varepsilon>V_{\mathrm{m}},\end{cases}
$$

where $T(\varepsilon)$ is a classical transit time of a particle between crystallographic planes. Hence the normalization constant is

$$
C_{1}=\frac{1}{\sqrt{1+|q(\varepsilon)|^{2}}} \sqrt{\frac{E_{0}}{T}} .
$$

If the quantum effects of tunnelling and abovebarrier reflection are neglected, then $|q|^{2}$ takes two values only : $|q|^{2}=0$, when $\varepsilon>V_{\mathrm{m}}$ and $|q|^{2}=1$ when $\varepsilon<V_{\mathrm{m}}$. Therefore the normalization constant has a jump discontinuity when $\varepsilon$ passes from belowbarrier range to above-barrier range. This is clarified under a classical approach to the considered problem : 


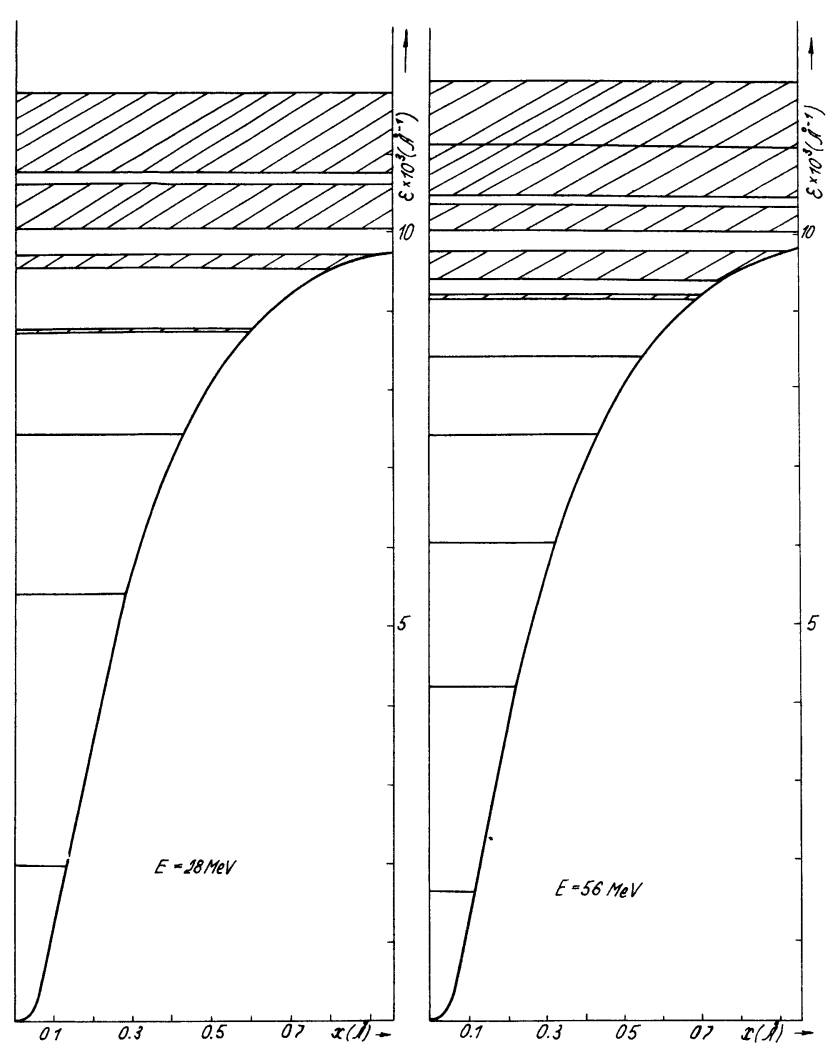

a
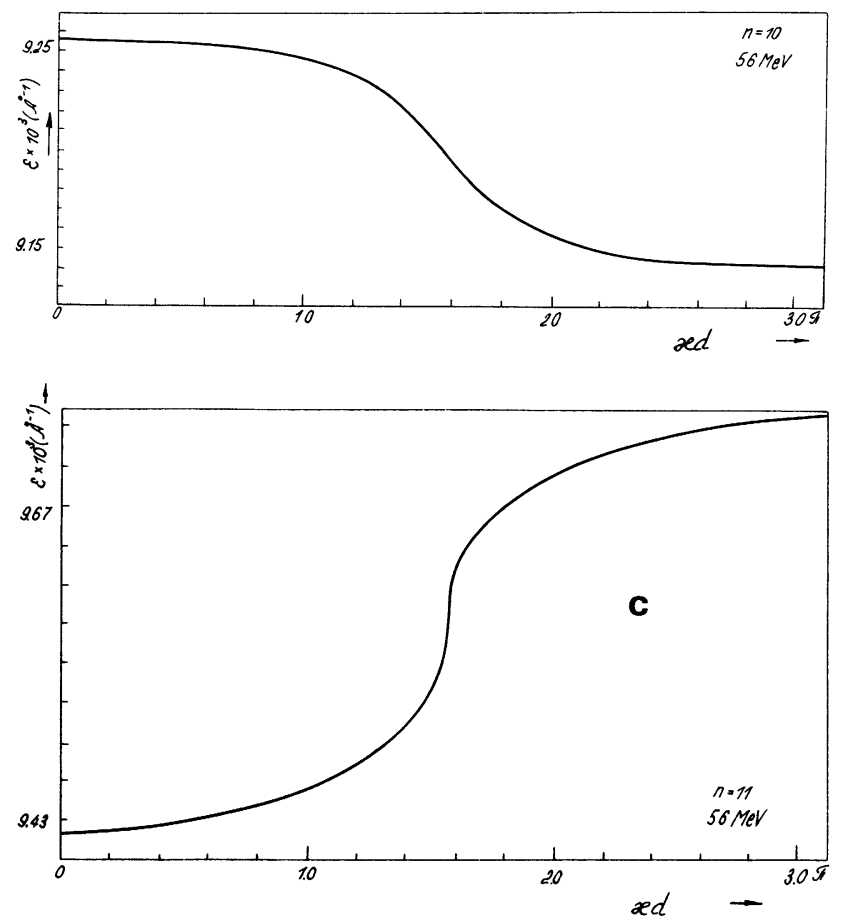

Fig. 1. - The characteristics of the band spectrum of the electrons and positrons channelled along the $(1,1,0)$ plane in $\mathrm{Si}$ crystal : a) disposition of zones for electrons with energy $E=28 \mathrm{MeV}$ (in the left) and $E=56 \mathrm{MeV}$ (in the right); the solid line is the Molière potential averaged over the thermal vibrations; energies in units $10^{8} \mathrm{~cm}^{-1}=2 \mathrm{keV}$; $b$ ) the same for positrons; dotted lines show the level disposition in the parabolic potential which is represented by dashed line ; $c$ ) the detailed structure of zones with $n=10$, 11 for positrons with $E=56 \mathrm{MeV}$.

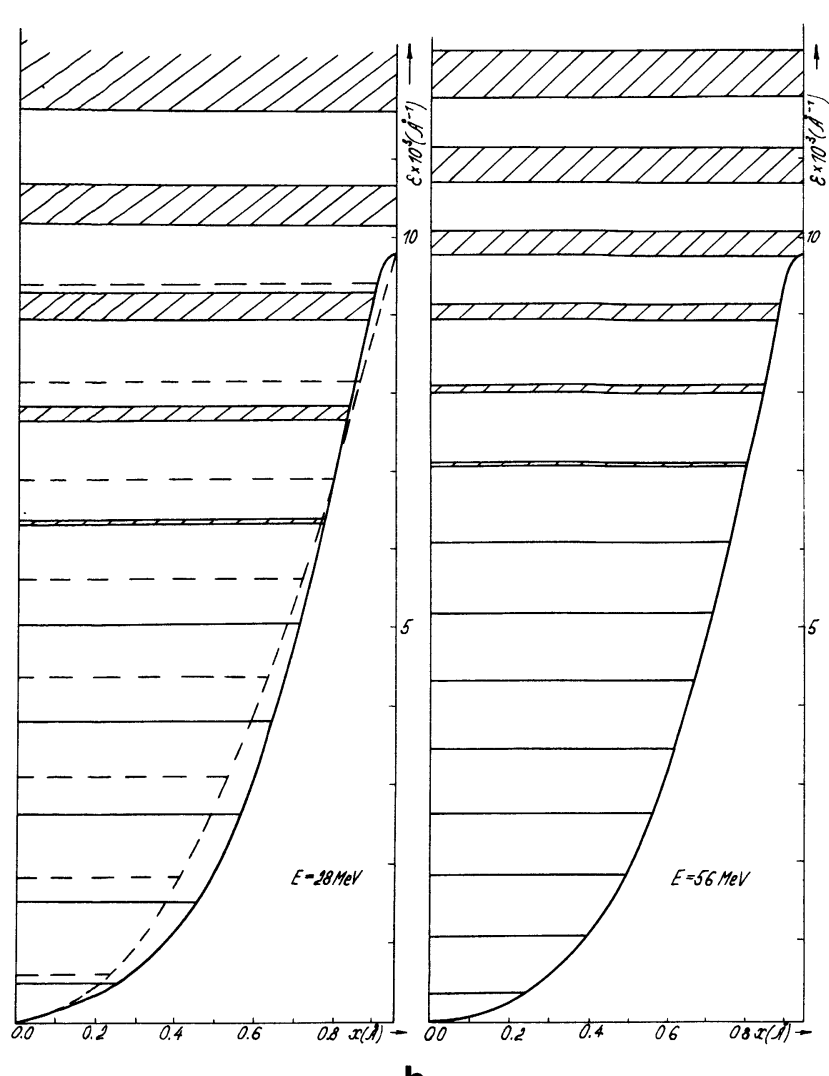

b

in this case the particle movement period tests the jump discontinuity too and as a result the radiation intensity and radiation spectrum from the particles with flying angle $\theta<\theta_{\mathrm{L}} \equiv \sqrt{\frac{2 V_{\mathrm{m}}}{E_{0}}}$ and with angle $\theta>\theta_{\mathrm{L}}$ sharply differ one from another. The artificial contrast of the radiation from the particle moved above the potential barrier and the radiation from the channelled particle arises under the classical consideration of this problem [20]. In fact from the quantum point of view both types of radiation have a mutual nature and are conditioned by the transitions between the energy bands of the particle transverse movement.

The formula (20) fails when the energy level closely equals the barrier potential and a classical period $2 T$ becomes infinite. However using the analytical solution (15) allows a regularization of the expression of the normalized integral (20). In fact, let us introduce some point $a$ belonging to the interval $\left(-\frac{d}{2} ; \frac{d}{2}\right)$ and meeting the conditions which are joint in a case when the particle movement is quasiclassical

$$
\frac{d}{2}-a \ll \frac{d}{2} ; \quad \sqrt[4]{4 \lambda}\left(\frac{d}{2}-a\right) \gg 1 .
$$

Then if the potential is symmetrical with respect to point $d / 2$ one can obtain the following formula using the asymptotic expression for $D_{m}(z)$ 


$$
\begin{gathered}
I=I_{p} \approx 2 \int_{x_{2}-a}^{x_{2}}|f|^{2} \mathrm{~d} x+2 \int_{0}^{x_{2}-a} \frac{\mathrm{d} x}{p(x)} \approx 2\left[C(\varepsilon) \int_{0}^{x_{2}}\left|D_{m}\left[x \sqrt[4]{4 \lambda} \exp \left(i \frac{\pi}{2}\right)\right]\right|^{2} \mathrm{~d} x+\right. \\
\left.+\int_{0}^{x_{2}}\left(\frac{1}{p(x)}-\frac{1}{p_{0}(x)}\right) \mathrm{d} x\right] \\
C(\varepsilon)= \begin{cases}\exp \left(-\frac{\tau_{1}}{4}\right) ; & \varepsilon<V_{\mathrm{m}} \\
\exp \left(-\frac{\tau_{2}}{4}\right) ; & \varepsilon>V_{\mathrm{m}}\end{cases} \\
p_{0}(x)=\sqrt{2 E_{0}\left(\varepsilon-V_{\mathrm{m}}+k x^{2}\right)}
\end{gathered}
$$

Taking into account the dispersion equation, one can transform the expression for $|q|^{2}$ as follows

$|q|^{2}= \begin{cases}\frac{A^{2}+1-2 \cos ^{2} \sigma-2 \sin \sigma \sqrt{A^{2}-\cos ^{2} \sigma}}{A^{2}+1} ; & \varepsilon<V_{\mathrm{m}} \\ \frac{B^{2}+1-2 \cos ^{2} \sigma_{0}-2 \sin \sigma_{0} \sqrt{B^{2}-\cos ^{2} \sigma_{0}}}{B^{2}+1} ; & \varepsilon>V_{\mathrm{m}},\end{cases}$

where

$$
A=\left(2 \mathrm{e}^{\tau_{1} / 2} \cosh \frac{\tau_{1}}{2}\right)^{-1} ; \quad B=\left(2 \mathrm{e}^{-\tau_{2} / 2} \cosh \frac{\tau_{2}}{2}\right)^{-1}
$$

The formulae (21) and (22) permit us to calculate the normalization constant $C_{1}$ for any allowable value of variable $\varepsilon$. The normalization constant $C_{1}$ behaviour is compared with the behaviour of the normalization constant without regularization in figure 2 .

It is to be noticed that there is the closed form expression for the integral of $\left|D_{m}\right|^{2}$ in (21). It is determined by the $G$-function of Majer [18]. However we calculated this integral numerically using the $D$-function integral representation.

Now we consider the calculation of the population coefficients of the energy levels when the defined form wave package falls on the crystal. For the right

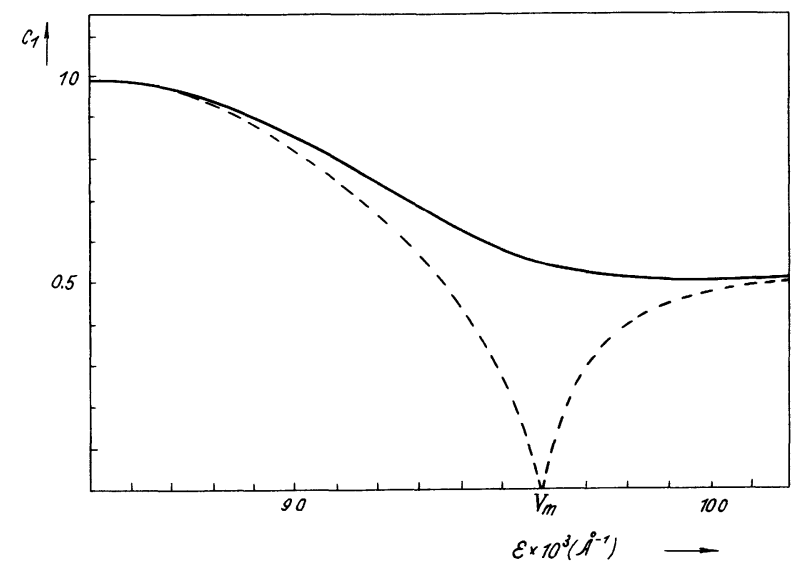

Fig. 2. - Comparison of the normalization constants without regularization (dotted line) and with regularization (solid line). interpretation of the radiation spectrum the detailed consideration of this point is very important. At first let us discuss the definition of the population coefficient and consider its dependence on the package configuration. Usually the incident beam is characterized by the angle $\theta$ only, whereas the population coefficient $C_{n \kappa}$ being defined by the expression

$$
Q_{n \kappa}=\left|C_{n \kappa}\right|^{2} ; \quad C_{n \kappa}=\int\left[\psi_{n \kappa}(\mathbf{r}) \psi_{0}(\mathbf{r})\right]_{z=0} \mathrm{~d} x \mathrm{~d} y
$$

depends on the configuration of the wave package $\psi_{0}$. However one can show that for real experiments this dependence is not important.

Let us decompose the function $\psi_{0}(\mathbf{r})$ in a Fourier integral :

$\psi_{0}(\mathbf{r})=\int a\left(\mathbf{p}-\mathbf{p}_{0}\right) \mathrm{e}^{i \mathbf{p r}} \mathrm{d} \mathbf{p} ; \int\left|a\left(\mathbf{p}-\mathbf{p}_{0}\right)\right|^{2} \mathrm{~d} \mathbf{p}=1$

where $a\left(\mathbf{p}-\mathbf{p}_{0}\right)$ is a function which has its maximum at the point $\mathbf{p}_{0}\left(p_{0 z} \theta ; 0 ; p_{0 z}\right)$ and which is nonzero in the range $\Delta p_{x}=\left|p_{x}-p_{0 x}\right| \sim \frac{1}{\Delta x}$ where $\Delta x$ is the width of the incident beam.

Substituting (24) in (22) and taking into account that in a case of real experiment $\Delta x$ is much greater than the distance between crystallographic planes we obtain that $Q_{n \kappa}$ are determined by the direction of momentum $\mathbf{p}_{0}$ only and are not dependent on the wave package configuration. Under the classical consideration of 
this problem the momentum $\mathbf{p}_{0}$ direction coincides with the particle motion direction.

Thus the population coefficients $Q_{n k}$ are expressed as follows

$$
Q_{n \kappa}=\left|C_{n \kappa}^{(0)}\right|^{2} ; \quad C_{n \kappa}^{(0)}=\int_{-d / 2}^{d / 2} \varphi_{n \kappa}^{*}\left(x, E_{0}\right) \mathrm{e}^{i p_{0_{z}} x \theta} \mathrm{d} x,
$$

where the index $\kappa$ is determined by the condition $\kappa=\left[p_{0 z} \theta-\frac{2 \pi l}{d}\right] ; \kappa$ is the crystal momentum of the considered one-dimensional crystal;

$$
E_{0}=\sqrt{p_{0 z}^{2}+m^{2}} .
$$

Let us take note that the momentum $p_{z}$, determining the total wave function (3) does not equal $p_{0 z}$ because the boundary continuity condition is used for the preassigned total energy. Therefore

$$
p_{z} \approx p_{0 z}+\left(\frac{p_{0 x}^{2}}{2 E_{0}}-\varepsilon_{n \kappa}\right) ; \quad p_{0 x}=p_{0 z} \theta .
$$

First of all let us consider the cases in which quantum effects are not important. Then

$$
\begin{aligned}
C_{n \kappa}^{(0)}=C_{1} \int_{-d / 2}^{d / 2} & \frac{\mathrm{d} x}{\sqrt{p(x)}} \times \\
& \times \exp \left[-i \int_{x_{1}}^{x} p\left(x^{\prime}\right) \mathrm{d} x^{\prime}+i p_{0 x} x\right] .
\end{aligned}
$$

Under the considered approximation, we use the sleepest descent method for calculating the integral (26) and as a result we obtain the expression which was first found in reference [21]

$$
\begin{gathered}
C_{n \kappa}^{(0)}=\frac{\sqrt{\pi} C_{1}}{\sqrt{E_{0}\left|V^{\prime}\left(x_{0}\right)\right|}} \exp \left[i p_{0 x} x_{0}-i \int_{x_{1}}^{x_{0}} p(x) \mathrm{d} x\right] \\
0<\varepsilon_{n \kappa}-\frac{p_{0 x}^{2}}{2 E_{0}}<V_{\mathrm{m}}
\end{gathered}
$$

where the saddle point $x_{0}$ is determined by the condition

$$
p_{0 x}=p\left(x_{0}\right) ; \quad V\left(x_{0}\right)=\varepsilon_{n \kappa}-\frac{p_{0 x}^{2}}{2 E_{0}} .
$$

In the cases both $\varepsilon_{n \kappa}-\frac{p_{0 x}^{2}}{2 E_{0}}>V_{\mathrm{m}}$ and $\varepsilon_{n \kappa}-\frac{p_{0 x}^{2}}{2 E_{0}}<0$ the equation (28) has no real roots (noting that the energy $\varepsilon$ counts off the potential minimum both for electrons and for positrons). But there is always the complex root of this equation that is $z_{0}=x_{0}+i y_{0}$. The nonzero imaginary part of the equation (28) root means an exponential decrease of the population coefficients in this energy range. Analytical continuation of the quasiclassical wave function permits us to obtain the following expressions for the population coefficients in the different energy ranges

$$
Q_{n \kappa}=\left\{\begin{array}{l}
\frac{\pi\left|C_{1}\right|^{2}}{E_{0}\left|V^{\prime}\left(x_{0}\right)\right|} ; \quad 0<\varepsilon_{n \kappa}-\frac{p_{0 x}^{2}}{2 E_{0}}<V_{\mathrm{m}} ; \\
\frac{\pi\left|C_{1}\right|^{2}}{E_{0}\left|V^{\prime}\left(z_{0}\right)\right|} \exp \left[-2 \int_{0}^{y_{0}} \sqrt{\left.2 E_{0}\left[\varepsilon_{n \kappa}-V\left(\frac{d}{2}+i y\right)\right] \mathrm{d} y+2 p_{0 x} y_{0}\right], \quad \varepsilon_{n \kappa}-\frac{p_{0 x}^{2}}{2 E_{0}}>V_{\mathrm{m}} ;}\right. \\
\frac{\pi\left|C_{1}\right|^{2}}{E_{0}\left|V^{\prime}\left(z_{0}\right)\right|} \exp \left[2 \int_{0}^{y_{0}} \sqrt{2 E_{0}\left[\varepsilon_{n \kappa}-V(i y)\right]} \mathrm{d} y-2 p_{0 x} y_{0}\right], \quad \varepsilon_{n \kappa}-\frac{p_{0 x}^{2}}{2 E_{0}}<0 .
\end{array}\right.
$$

A complex point of turn $z_{0}$ is determined by the equality

$$
\varepsilon_{n \kappa}-\frac{p_{0 x}^{2}}{2 E_{0}}=V\left(z_{0}\right)
$$

under any $p_{0 x}$.

When the saddle point is in the neighbourhood of the barrier potential point or the potential minimum point, the expression (29) becomes invalid. However in the neighbourhood of these points the real potential is approximated by a parabola and in these energy ranges $Q_{n \kappa}$ is calculated using analytical solution of the Schrödinger equation (1)

$$
\begin{gathered}
\varphi_{1}=A_{1}\left[D_{m_{1}}\left(x \sqrt[4]{4 \lambda_{1}}\right)+D_{-m_{1}-1}\left(i x \sqrt[4]{4 \lambda_{1}}\right)\right] \\
\varepsilon_{n \kappa} \approx \frac{p_{0 x}^{2}}{2 E_{0}} \\
\varphi_{2}=A_{2} D_{m_{2}}\left[x_{1} \exp \left(-i \frac{\pi}{4}\right) \sqrt[4]{4 \lambda_{2}}\right] \\
\varepsilon_{n \kappa}-\frac{p_{0 x}^{2}}{2 E_{0}} \approx V_{\mathrm{m}}
\end{gathered}
$$

where

$$
m_{1}=-\frac{1}{2}+i \frac{\sqrt{\lambda_{1}}}{2} \frac{\left(\varepsilon-V_{\mathrm{m}}\right)}{K_{1}}
$$




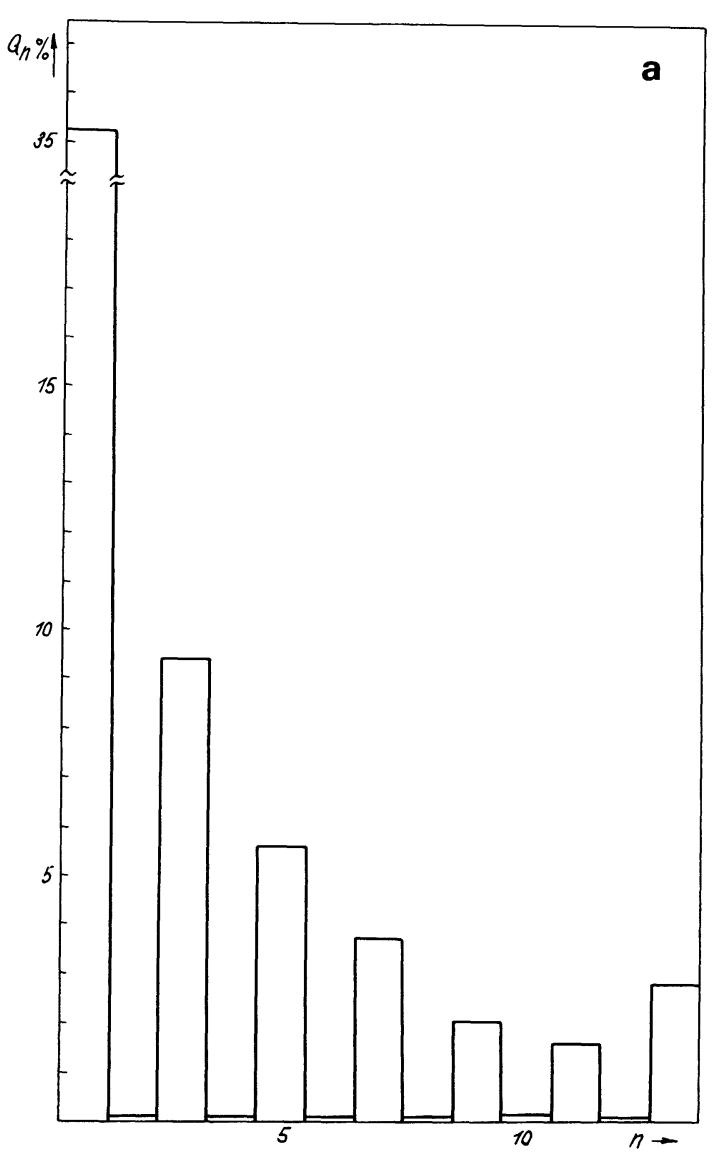

Fig. 3. - The band population coefficients for $p_{x}=0$ : a) positrons with energy $E=56 \mathrm{MeV} ; b$ ) the same for electrons.

$$
\begin{gathered}
m_{2}=-\frac{1}{2}+\frac{\sqrt{\lambda_{2}}}{2} \frac{\varepsilon}{K_{2}} ; \quad \lambda_{1,2}=\sqrt{2 E_{0} K_{1,2}} \\
K_{1}=\frac{1}{2}\left|V^{\prime \prime}(0)\right| ; \quad K_{2}=\frac{1}{2}\left|V^{\prime \prime}\left(\frac{d}{2}\right)\right| .
\end{gathered}
$$

Coefficients of linear combination of the parabolic cylinder functions in (30) are chosen from the condition that for $\left|m_{1,2}\right| \gg 1$ the functions $\varphi_{1,2}$ coincide with quasiclassical solutions.

The integral (26) determining the coefficient $C_{n k}^{(0)}$ can be turned into the infinite integral as the integration in the neighbourhood of points $x \approx 0$ and $x \approx d / 2$ brings the main contribution to this integral. As a result, the coefficients may be expressed over special functions but this expression is sufficiently

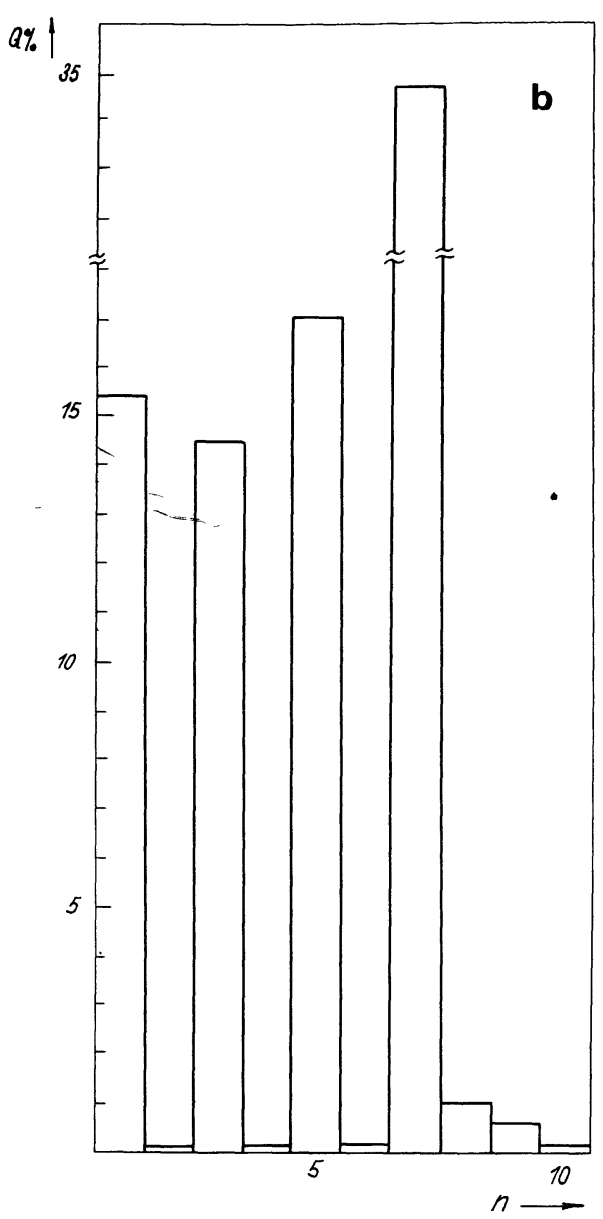

complicated. Therefore we found the population coefficients by calculating the integral in (26) numerically. Figure 3 shows the behaviour of the population coefficients both for electrons and for positrons for monochromatic beams. In both these cases the behaviour of the population coefficients is essentially different and this fact must be accounted for in calculating the channelled particle radiation.

In the real experiment particles in the beam have a sufficiently large angle dispersion $\Delta \theta$ and it is necessary to average $Q_{n \kappa}$ over these angles. If $\Delta \theta$ is the same order as $\theta_{\mathrm{L}}=\sqrt{\frac{2 V_{\mathrm{m}}}{E}}$ one can find the simple formula for the averaged population coefficients on the basis of quasiclassical expression (29). The formula is

$$
\bar{Q}_{n \kappa}=\left|C_{n \kappa}\right|^{2} \cdot 4 \pi\left[\int_{0}^{x} \frac{a\left(p_{x}-p_{0 x}\right)}{\sqrt{2 E_{0}\left[\varepsilon_{n \kappa}-V(x)\right]}} \mathrm{d} x+\int_{0}^{x} \frac{a\left(p_{x}+p_{0 x}\right)}{\sqrt{2 E_{0}\left[\varepsilon_{n \kappa}-V(x)\right]}} \mathrm{d} x\right] ; \quad p_{x}=\sqrt{2 E_{0}\left[\varepsilon_{n \kappa}-V(x)\right]},
$$

where $a\left(p_{x}-p_{0 x}\right)$ is the function describing the particle angle distribution in the beam. The normalization condition is

$$
\sum_{n} \int_{-\pi / d}^{\pi / d} \bar{Q}_{n \kappa} \mathrm{d} \kappa=1
$$


4. A zone spectrum «scaling ». - The peculiar character of the problem of zone spectrum building for ultrarelativistic channelled particles is that the longitudinal energy enters as a parameter in the equation defined the particle transverse movement. Besides the interplane potential parameters essentially change when the crystallographic plane and material of the crystal where the particle is channelled are changed. Therefore it seems at first sight that it is necessary to calculate independently a zone spectrum for any modification of the experimental conditions. But it is proved that the dependence of zone spectrum characteristics from $E_{0}$, amplitude $V_{\mathrm{m}}$ and period of the interplane potential is universal in the limits of the considered method. This circumstance permits us to make a " scaling " of zone spectrum and to find the values $\varepsilon_{n \kappa}$ for different $E_{0}, V_{\mathrm{m}}$ and $d$ on the basis of sole " calibrating " spectrum.

Indeed, let us consider the equation which defines the values corresponding to the centre of zones

$$
\int_{a}^{b} \sqrt{2 E_{0}[\varepsilon(n)-V(x)]} \mathrm{d} x=\pi\left(n+\frac{1}{2}\right),
$$

where $a=x_{1}, b=x_{2}$ for below-barrier region of $\varepsilon$ and $a=-d / 2, b=d / 2$ for $\varepsilon>V_{\mathrm{m}}$.

An analogous equation but for the other energy $E_{1}$ and for the potential $V(x)=\lambda V(\alpha x)$ is

$$
\int_{a_{1}}^{b_{1}} \sqrt{2 E_{01}\left[\varepsilon\left(n_{1}\right)-\lambda V(\alpha x)\right]} \mathrm{d} x=\pi\left(n_{1}+\frac{1}{2}\right) .
$$

Let us consider the numbers $n$ and $n_{1}$ as continuous variables. Then it follows directly from equations (32) and (33) that $\varepsilon(n)$ and $\varepsilon_{1}\left(n_{1}\right)$ connect by means of the correlation

$$
\varepsilon_{1}\left(n_{1} ; E_{01} ; \lambda ; \alpha\right)=\lambda \varepsilon\left(\frac{n}{\sqrt{\lambda}} \alpha \sqrt{\frac{E_{0}}{E_{01}}}\right) .
$$

Now suppose that the "calibrating " spectrum $\varepsilon(n)$ is known. Then the discrete energy levels $\varepsilon_{1}\left(n_{1}\right)$ for arbitrary $E_{1} ; \alpha ; \lambda$ and for natural $n_{1}$ one can find by means of the formula which is just with an accuracy of the quasiclassical parameter

$$
\begin{aligned}
\varepsilon_{1}\left(n_{1}\right)=\lambda \varepsilon\left(n_{-}\right)+\left[\alpha n_{1}\right. & \left.\sqrt{\frac{E_{0}}{E_{01} \lambda}}-n_{-}\right] \times \\
& \times \lambda\left[\varepsilon\left(n_{+}\right)-\varepsilon\left(n_{-}\right)\right],
\end{aligned}
$$

where $n_{+}$and $n_{-}$are the natural parts of the number $\alpha n_{1} \sqrt{\frac{E_{0}}{E_{01} \lambda}}$ calculated with surplus and scarcity accordingly.

In the above-barrier region where the width of a forbidden zone is exponentially small the dispersion equation (19) is simplified

$$
\cos \kappa d \simeq \cos \sigma_{2}(\varepsilon)
$$

and the energy levels $\varepsilon_{1}\left(n_{1} ; \kappa\right)$ are expressed from the " calibrating " spectrum $\varepsilon(n ; \kappa)$ as follows

$$
\varepsilon_{1}\left(n_{1} ; \kappa\right) \simeq \lambda \varepsilon\left(\alpha n_{1} \sqrt{\frac{E_{0}}{E_{01} \lambda}}+\Delta n ; \tilde{\kappa}\right) .
$$

The quasimomentum $\tilde{\kappa}$ and the value $\Delta n$ are defined by following inequality

$$
\left|\alpha \sqrt{\frac{E_{0}}{E_{01} \lambda}} \kappa-\frac{2 \pi \Delta n}{d}\right| \leqslant \frac{\pi}{d} .
$$

In order to improve the accuracy of the correlation (36) one can use interpolating formula analogies (35).

And after all let us consider the essentially quantum region of the value $\varepsilon \approx V_{\mathrm{m}}$ when the width of forbidden zone is compared with the width of allowed zone and the dispersion equation is sufficiently complicate. But it remains to take into account that in this region the potential $V(x)$ coincides with parabola

$$
V(x) \approx V_{\mathrm{m}}-k x_{1}^{2}
$$

and the equation (19) does not change under the following transformation

$$
\Delta \varepsilon\left(n_{1} ; \kappa\right)=\alpha \sqrt{\frac{\lambda E_{0}}{E_{01}}} \Delta \varepsilon\left(\alpha n_{1} \sqrt{\frac{E_{0}}{E_{01}}} ; \kappa\right),
$$

where the value $\Delta \varepsilon(n ; \kappa)$ was defined earlier.

The totality of the formulae (36)-(37) permits us to built a zone energy spectrum of channelled particle with an accuracy $\lesssim 2 \%$ for different values of the total energy $E \gtrsim 10 \mathrm{MeV}$ and for different potential parameters if zone spectrums described in $\S 3$ are used as " calibrating " spectrums.

And in conclusion of this paragraph we note that the considered method of the spectrum "scaling " may be used in the case of axis channelling and for channelled ions. Besides a zone spectrum " scaling" permits us to make the analytical transformation of radiation spectra from the channelled particles when the experimental conditions change.

5. Conclusions. - The investigation carried out in our work shows that the use of the semiclassical approximation enables us to calculate all the characteristics of the channelled electrons and positrons in the whole range of the transverse movement energy. The obtained results are significant for the quantitative falculation of the channelled particle radiation spectrum in the crystal. This problem will be considered separately.

Acknowledgments. - We are very greatful Prof. V. G. Baryshevsky for stimulating discussions and continuing interest in our work. 


\section{References}

[1] Tomson, M., Usp. Fiz. Nauk 99 (1969) 297.

[2] Kalashnikov, N. P., Koptelov, E. A., Ruazanov, M. I., Fiz. Tverd. Tela 14 (1972) 1211.

[3] Vorobiev, A. A., Kaplin, V. V., Vorobiev, S. A., Nucl. Instrum. Methods 127 (1975) 265.

[4] Baryshevsky, V. G., Dubovskaya, I. Ya., Abstr. VIII Conf. of Charged Particle Collision in Crystal, Moscow State University (1976) 51.

[5] Kumakhov, M. A., Phys. Lett. A 57 (1976) 17.

[6] Vorobiev, S. A. et al., Zh. Eksp. Teor. Fiz. Pisma 29 (1979) 414.

[7] Miroshnichenko, K. N., Miorry, D. D., Avakyan, R. O., Zh. Eksp. Teor. Fiz. Pisma 29 (1979) 786.

[8] Swent, R. L., Pantell, R. N., Alguard, M. J., Nucl. Instrum. Methods 170 (1980) 7.

[9] Basylev, V. A., Glebov, V. I., Zhevago, N. K., Zh. Eksp. Teor. Fiz. 78 (1980) 62.

[10] Baryshevsky, V. G., Dubovskaya, I. Ya., Phys. Status Solidi (b) 82 (1977) 403.

[11] Baryshevsky, V. G., Grubich, A. O., Dubovskaya, I. Ya., Phys. Status Solidi (b) 88 (1978) 351.
[12] Chevganov, B. A., Feranchuk, I. D., Abstr. XI Conf. of Charged Particle Collision in Crystal, Moscow State University (1981) 74.

[13] Pokrovsky, V. L., Khalatnikov, I. M., Zh. Eksp. Teor. Fiz. 40 (1961) 1713.

[14] Diknne, A. M., Zh. Eksp. Teor. Fiz. 40 (1961) 1423.

[15] Tulupov, A. V., Radiat. Eff. 56 (1981) 77.

[16] Kagan, Yu., Kononetz, Yu., Zh. Eksp. Teor. Fiz. 58 (1970) 226.

[17] FoK, V. A., The Problems of the Diffraction and Electromagnetic Wave Propagation, Moscow (1970) 347.

[18] Bateman, H., Erderyi, A., Higher Transcendental Functions (McGraw-Hill Book Company Inc.) 1955, vol. 3.

[19] Gemmell, D. S., Rev. Mod. Phys. 46 (1974) 129.

[20] Podgoretzky, M. I., Yadernaya Fiz. 31 (1980) 417.

[21] Ryabov, V. A., Zh. Eksp. Teor. Fiz. 67 (1974) 150. 